\title{
Cluster observation of few-hour-scale evolution of structured plasma in the inner magnetosphere
}

\author{
M. Yamauchi ${ }^{1}$, I. Dandouras ${ }^{2,3}$, H. Rème ${ }^{2,3}$, R. Lundin ${ }^{4}$, and L. M. Kistler ${ }^{5}$ \\ ${ }^{1}$ Swedish Institute of Space Physics, P.O. Box 812, 98128 Kiruna, Sweden \\ ${ }^{2}$ CNRS, Institut de Recherche en Astrophysique et Planetologie (IRAP), BP 44346, 31028, Toulouse cedex 4, France \\ ${ }^{3}$ University of Toulouse, UPS-OMP, IRAP, Toulouse, France \\ ${ }^{4}$ Swedish Institute of Space Physics, Umeå, Sweden \\ ${ }^{5}$ University of New Hampshire, Durham, NH, USA \\ Correspondence to: M. Yamauchi (m.yamauchi@irf.se)
}

Received: 15 March 2013 - Revised: 21 July 2013 - Accepted: 22 July 2013 - Published: 12 September 2013

\begin{abstract}
Using Cluster Ion Spectrometry (CIS) data from the spacecraft-4 perigee traversals during the 2001-2006 period (nearly 500 traversals after removing those that are highly contaminated by radiation belt particles), we statistically examined the local time distribution of structured trapped ions at sub- to few-keV range as well as inboundoutbound differences of these ion signatures in intensities and energy-latitude dispersion directions. Since the Cluster orbit during this period was almost constant and approximately north-south symmetric at nearly constant local time near the perigee, inbound-outbound differences are attributed to temporal developments in a $1-2 \mathrm{~h}$ timescale. Three types of structured ions at sub- to few $\mathrm{keV}$ range that are commonly found in the inner magnetosphere are examined:
\end{abstract}

1. Energy-latitude dispersed structured ions at less than a few $\mathrm{keV}$,

2. Short-lived dispersionless ion stripes at wide energy range extending $0.1-10 \mathrm{keV}$,

3. Short-lived low-energy ion bursts at less than a few hundred $\mathrm{eV}$.

The statistics revealed that the wedge-like dispersed ions are most often observed in the dawn sector $(60 \%$ of traversals), and a large portion of them show significant enhancement during the traversals at all local times. The short-lived ion stripes are predominantly found near midnight, where most stripes are significantly enhanced during the traversals and are associated with substorm activities with geomagnetic
$\mathrm{AL}<-300 \mathrm{nT}$. The low-energy bursts are observed at all local times and under all geomagnetic conditions, with moderate peak of the occurrence rate in the afternoon sector. A large portion of them again show significant enhancement or decay during the traversals.

Keywords. Magnetospheric physics (energetic particles, trapped)

\section{Introduction}

Trapped hot ions in the inner magnetosphere ( $L<7$ is considered in this paper) bounce between the Northern Hemisphere and the Southern Hemisphere with short bounce periods of only around $10 \mathrm{~min}$ for $100 \mathrm{eV}$ protons and $1 \mathrm{~min}$ for $10 \mathrm{keV}$ protons at $L=4$ (see, e.g., Quinn and McIlwain, 1979). Therefore, for any phenomena with longer timescales than a few tens of minutes in the sub-auroral region, the ion population of $>100 \mathrm{eV}$ in the inner magnetosphere is expected to be the same between the conjugate regions, i.e., at a fixed latitude (or $L$ value) and longitude. In other words, one can ignore the hemispheric difference when examining the temporal changes of intensities or latitudinal profiles of these ion populations.

Cluster perigee traversals in the inner magnetosphere during the 2001-2006 period have a perigee altitude of 4 Earth radii at the equator, and the orbit is nearly symmetric about the equatorial plane along nearly the same longitude, with less than $1-2 \mathrm{~h}$ local time difference between the inbound and the outbound passes. The symmetry with respect to the 


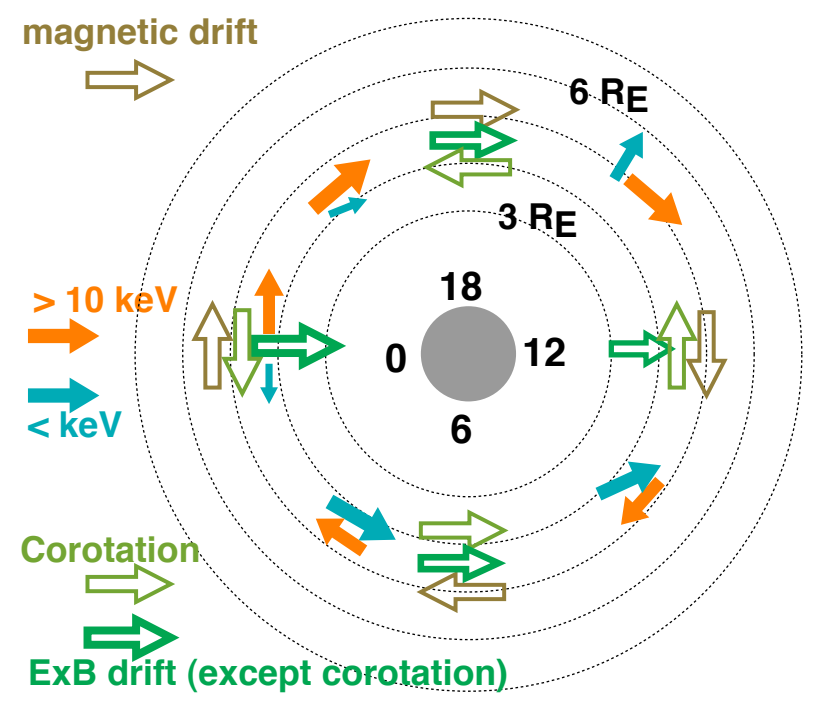

Fig. 1. Illustration of ion drift in the inner magnetosphere. The three major components of the drift (magnetic drift, co-rotation, and $\boldsymbol{E} \times \boldsymbol{B}$ drift) are given by the empty arrows, and the resultant ion velocities are given by the solid arrows for high-energy and lowenergy components under a moderate magnetospheric condition. The velocity is schematically represented by the size of the arrow. Due to the temporal variation of the electric field, the drift motion of sub-keV ions changes dynamically.

equatorial plane for both the orbit and the motion of trapped ions allows us to interpret the inbound-outbound differences in the latitudinal profile of the ion population as temporal changes by, for example, ion energization, transport, or loss, with a timescale on the order of $1 \mathrm{~h}$. If an ion signature is energy-latitude dispersed, this difference should appear as an inbound-outbound asymmetry in the ion dispersion direction with respect to the equator because the latitude can be replaced by the distance from the equatorial plane. Furthermore, the nearly constant orbital parameters during the 2001-2006 period allow us to compare simply the different traversals to obtain statistics of the different ion populations to determine their local time distribution and correlation with substorm activities.

Yamauchi et al. (2006) showed one example of a clear inbound-outbound difference at around 09 MLT (magnetic local time). In that event, drifting protons from an earlier local time and oxygen outflow from the ionosphere suddenly appeared during the outbound pass in the northern magnetosphere, although no signature was observed during the inbound pass along the geomagnetically conjugate region with the inbound pass. This observation indicated a temporal change in the ion population in the inner magnetosphere, and it turned out to be related to a substorm onset. The obvious next step is to perform a statistical study of similar kinds of asymmetries.

Such a work requires a good classification of the latitudinal ion profiles into several "basic energy-latitude patterns" of ion populations coming from specific source regions via specific transport mechanisms and elapsed times, from $10 \mathrm{~min}$ to $10 \mathrm{~h}$ (Horwitz and Chappell, 1979; Ejiri et al., 1980; Olsen et al., 1987; Ebihara et al., 2001; Yamauchi and Lundin, 2006; Yamauchi et al., 2009b). However, this task is not simple because the observed ion population of $<$ several $\mathrm{keV}$ in the inner magnetosphere is a convolution of various ion populations, which are sensitive to changes in the drift direction, location, and magnetospheric activity.

The ion drift motion in the inner magnetosphere is generally determined by the energy-dependent magnetic (gradientB and curvature) drift, the energy-independent $\boldsymbol{E} \times \boldsymbol{B}$ drift, and co-rotation, where $\boldsymbol{E}$ is the externally imposed electric field. The magnetic drift and co-rotation drive ions westward and eastward, respectively, at all local times (LTs) in a nearly time-independent manner, while $\boldsymbol{E} \times \boldsymbol{B}$ drift driven by the time-dependent dawn-to-dusk electric field moves the drifting ions mainly sunward, i.e., westward in the evening sector and eastward in the morning sector (Alfvén and Fälthammar, 1963; Lennartsson et al., 1979), as illustrated in Fig. 1.

Due to the energy-dependence of the magnetic drift, the magnetic drift exceeds the co-rotation for $>10 \mathrm{keV}$ ions and vice versa for cold ions at all LTs. Since a balance of the above three drift components determines the ion drift motion, the time-dependent $\boldsymbol{E} \times \boldsymbol{B}$ drift controls the balancing energy that demarcates the westward drift (high energy) and the eastward drift (low energy). Due to the local time dependencies of the azimuthal component of the $\boldsymbol{E} \times \boldsymbol{B} \mathrm{drift}$ (cf. Fig. 1), this demarcation energy decreases as the local time increases from the morning sector to the evening sector (Quinn and McIlwain, 1979; Yamauchi et al., 2006). According to Kovrazhkin et al. (1999), the statistical demarcation energy ("ion gap" in their terminology) in the morning sector at $65^{\circ}$ invariant latitude $(L=5.6)$ is $5-8 \mathrm{keV}$. Below this demarcation energy, the balance of different drift components makes the final eastward drift even slower than the drift velocity of the dominating drift component and slower than the drift velocity of cold plasmaspheric ions. Therefore, the inbound-outbound differences of these low-energy ion patterns in the Cluster data indicate significant temporal changes of the source of these low-energy ions.

Previously, the four most common ion patterns at the energy range that corresponds to eastward drift in the Cluster Ion Spectrometry (CIS) data have been identified:

a. Plasma sheet-like ions: They appear at a wide latitudinal range over a limited energy range (horizontal bandlike structure in the spectrogram) below the ion gap that demarcates eastward and westward drift, i.e., in the eastward drifting domain (Woch and Lundin, 1993; Yamauchi et al., 2006). This is sometimes referred to as a "nose" (Vallat et al., 2007), but it is different from the classic "nose" structure in the westward drifting energy domain (e.g., Ejiri et al., 1980; Sheldon and Spence, 1997; Ganushkina et al., 2000). 
(a) CIS/CODIF (SC-4) , 2004-2-6

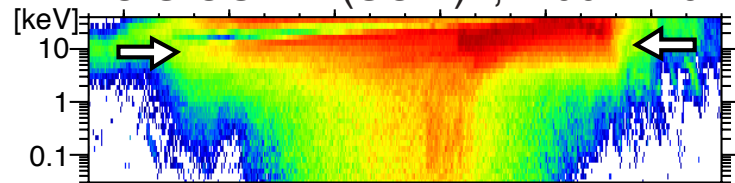

$\begin{array}{lllll} & \text { UT 09:30 10:00 10:30 11:00 11:30 } & 12: 00\end{array}$

\begin{tabular}{|c|c|c|c|c|c|c|}
\hline $\begin{array}{lc}\text { Dist } & 5.6 \\
\text { lLat } & -70.1 \\
\text { MLT } & 2.3 \\
\text { Xgse }-2.2 \\
\text { Ygse }-3.0 \\
\text { Zqse }-4.1\end{array}$ & $\begin{array}{c}5.1 \\
-66.3 \\
2.2\end{array}$ & $\begin{array}{l}4.7 \\
-62.8 \\
2.1\end{array}$ & $\begin{array}{r}4.2 \\
61.0 \\
2.0 \\
-3.7 \\
-2.2 \\
-0.7\end{array}$ & $\begin{array}{l}4.2 \\
61.8 \\
1.8\end{array}$ & $\begin{array}{l}4.2 \\
66.0 \\
1.5\end{array}$ & $\begin{array}{r}4.4 \\
72.2 \\
1.1 \\
-3.3 \\
-0.4 \\
2.9\end{array}$ \\
\hline
\end{tabular}

(b) CIS/CODIF (SC-4) , 2004-9-14

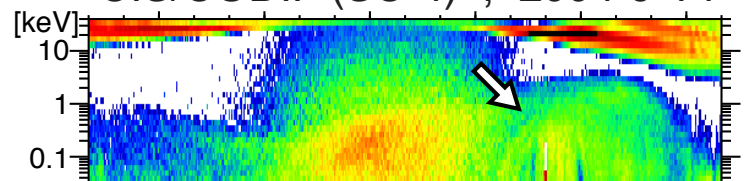

UT $\quad 00: 30 \quad 01: 00 \quad 01: 30 \quad 02: 00 \quad 02: 30$ 03:00

$\begin{array}{lccccccc}\text { Dist } & 5.2 & 4.8 & 4.5 & 4.4 & 4.4 & 4.5 & 4.8 \\ \text { ILat }-70.1 & -66.0 & -62.4 & 60.2 & 62.2 & 67.0 & 72.8 \\ \text { MLT } 12.2 & 11.9 & 11.7 & 11.6 & 11.5 & 11.4 & 11.3 \\ \text { Xgse 4.1 } & & & 4.3 & & & 2.5 \\ \text { Ygse 0.9 } & & & -0.6 & & & -1.8 \\ \text { Zgse -3.1 } & & & 0.4 & & & 3.7\end{array}$

(d) CIS/CODIF (SC-4) , 2002-8-8

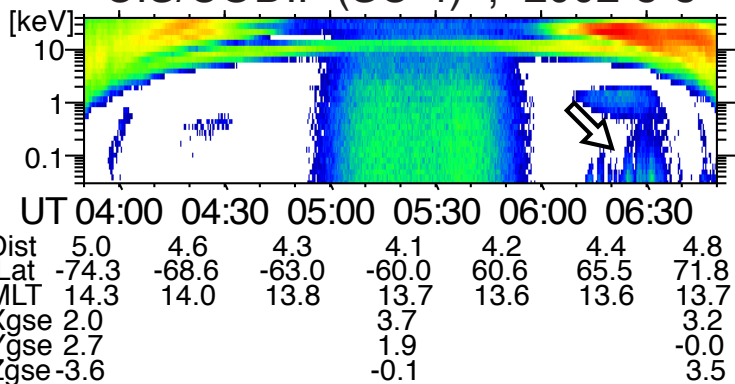

(e) CIS/CODIF (SC-4) , 2002-9-8

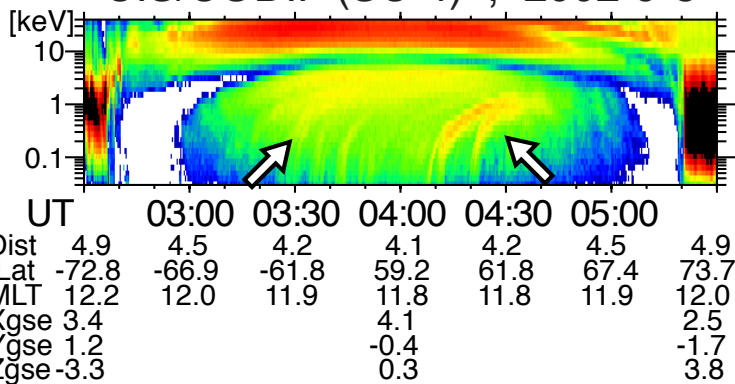

(c) CIS/CODIF (SC-4) , 2003-3-15

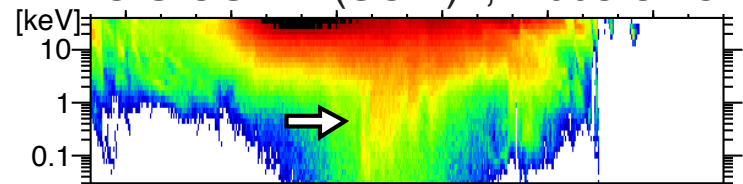

UT 01:30 02:00 02:30 03:00 03:30 04:00

$\begin{array}{lllllll}\text { Dist } 5.2 & 4.8 & 4.4 & 4.1 & 4.0 & 4.2 & 4.5 \\ \text { ILat -70.2 } & -65.0 & -60.5 & 63.3 & 66.3 & 72.9 & 80.6 \\ \text { MLT 22.9 } & 23.1 & 23.2 & 23.3 & 23.4 & 23.4 & 23.3 \\ \text { Xgse -3.2 } & & & -4.0 & & & -2.6 \\ \text { Ygse-1.1 } & & & 0.5 & & & 1.8 \\ \text { Zgse -4.0 } & & & -0.5 & & & 3.1\end{array}$

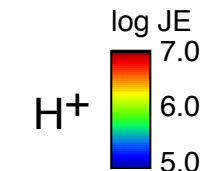

(keV cm-2 s-1 keV-1)

Fig. 2. Energy-time spectrograms of differential energy flux $\left(\mathrm{keV} \mathrm{cm}^{-2} \mathrm{~s}^{-1} \mathrm{str}^{-1} \mathrm{keV}^{-1}\right)$ of protons observed by CIS/CODIF on board Cluster SC-4 during perigee traversal on (a) 6 February 2004 (plasma sheet-like banded ions), (b) 14 September 2004 (wedge-like dispersed ions), (c) 5 March 2003 (vertical stripes), (d) 8 August 2002 (short burst of low-energy ions), and (e) 8 September 2002 (internal dispersive stripes). The object ion signature is marked by the empty arrow in each panel.

b. Wedge-like energy-latitude dispersed structured ions at sub-keV range (Yamauchi et al., 1996; Ebihara et al., 2001): These ions are found even at low altitude (Sauvaud et al., 1981; Newell and Meng, 1986). The energy of the source population is much lower than that of the first population, and two possible origins have been proposed for this population: a nightside plasma sheet that contains cold plasma of ionospheric origin (Yamauchi et al., 2009b; Engwall et al., 2009) and a direct supply from the ionosphere (Giang et al., 2009). It is not clear whether these ions are the same as sub-keV ions flowing in nearly the field-aligned directions as observed by many equatorial satellites (e.g., Fennel et al., 1981). Even if they are the same, we cannot simply assume a direct ionospheric origin as the past equatorial observations suggested, because ions with low-altitude mirror points are observed as nearly field-aligned near the equatorial plane.

c. A short-lived burst of ions extending from sub-keV to about $10 \mathrm{keV}$ (McIlwain, 1975; Yamauchi et al., 2006): The ion pattern is similar to those of the wedge-like dispersed ions but the dispersion is weak (nearly vertical in the spectrogram) and the energy range is wide. This signature is often associated with the so-called injection edge (e.g., McIlwain, 1974; Ejiri et al., 1980), and is most likely formed in a pulse-like manner during substorms. The origin of these ions is either the nightside plasma sheet or the ionosphere. 
d. Short bursts of low-energy ions that are isolated from the above structures: They have a peak energy flux at less than $100 \mathrm{eV}$, but at higher energy than plasmaspheric ions (Yamauchi et al., 2009a). The majority of the events are flowing in nearly field-aligned directions (Yamauchi et al., 1996, 2009a). The association with field-aligned warm ions at the equator (e.g., Horwitz and Chappell, 1979) is not clear.

Figures $2 \mathrm{a}-\mathrm{d}$ show examples of these ion populations. The individual signatures are marked by the empty arrows. Although these hot ions are expected to be observed in similar ways in the conjugate hemispheres (i.e., symmetric with respect to the equator), we selected in Fig. 2 the traversals when the ion populations are significantly different between the inbound and the outbound passes during Cluster perigee traversals. Figure 2a shows sudden intensification of the plasma sheet-like ions at a limited energy range in the eastward drifting domain (we call this "plasma sheet-like banded ions"). Figure $2 \mathrm{~b}$ shows sudden appearance of wedge-like energy-latitude dispersed ions (we call this "wedge-like dispersed ions" hereafter). Figure $2 \mathrm{c}$ shows sudden appearance of short-lived burst of ions covering a wide energy (we call these "vertical stripes" hereafter), and Fig. $2 \mathrm{~d}$ shows a short burst of low-energy ions.

Due to the energy-dependent drift velocity, the energylatitude dispersion of nearly azimuthally drifting ions becomes sharper with longer elapsed time, i.e., from nearly vertical stripes in the spectrogram like the third type (vertical stripes) to curved stripes like the second type (wedgelike dispersed ions) after some drift time and drift distance (Ebihara et al., 2001). However, we keep the second type and the third type as two different categories in the present study because the third type and the second type often coexist in an independent manner (Yamauchi et al., 2006), indicating that the third type may have different sources than the second type.

The energy-latitude dispersion also appears in the first category (plasma sheet-like banded ions), with lower energy at higher $L$ after a long elapsed time (Vallat et al., 2007). This feature can be attributed to different magnetic drift velocities at different $L$ shells (Ebihara et al., 2004), and can be distinguished from the wedge-like dispersed ions. However, the injection front of this population also shows energy-time or energy-latitude dispersions, and these ions are no longer distinguished from the wedge-like dispersed ions in both morphology and theory (McIlwain, 1974; Yamauchi et al., 1996; Ebihara et al., 2001).

As mentioned above, the threshold energy that demarcates the westward drift and the eastward drift decreases as the local time increases. During substorms, this demarcation energy increases due to the enhanced electric field (Yamauchi et al., 2009b). In this paper, however, we do not include the change in the ion energy in defining the inbound-outbound differences because we look for the sudden appearance/disappearance of an ion population rather than change in the drift velocity.

We statistically obtain the local time distribution of these ion signatures observed by Cluster during the 2001-2006 period. We also examine the inbound-outbound differences or asymmetry in the dispersion direction of these ion populations. We use CIS COmposition DIstribution Function (CODIF) instrument that covers $0.03-40 \mathrm{keV}$ (Rème et al., 2001). The double-coincidence detection for mass separation for CODIF removes a large part of contamination by the radiation belt particles inside the geosynchronous orbit. However, one-third of the traversals during the 2001-2006 period could not be used because of this type of severe contamination. Due to degradation of microchannel plates (MCPs), the sensitivity of CODIF started to differ significantly between different spacecraft (SC) after fall 2002 (Fig. 5 of Kistler et al., 2013). Therefore, we use only SC-4 in this statistical work.

\section{Statistics}

For the ion populations that are shown in Figs. 2a-d, we take statistics of (b)-(d) types. We examine both total observation frequency and the observation frequency of cases that show clear inbound-outbound differences (i.e., strong intensification or decay in the intensity). As one form of inboundoutbound difference, we also examine the energy-latitude dispersion direction for the second population (wedge-like dispersed ions): we examine (e) internal stripes that have energy-latitude dispersions asymmetric with respect to the equator as shown in Fig. 2e. This category is sometimes difficult to distinguish from the strong intensification of the wedge-like dispersed ions, but such cases are the minority.

We do not study the plasma sheet-like banded ions (Fig. 2a) because its energy-time pattern varies considerably over a wide energy range for both energy bands (narrow band to very wide band) and low-energy cutoff (from about $1 \mathrm{keV}$ to more than $10 \mathrm{keV}$ ). This variability makes it difficult to take statistics, particularly when we have to distinguish it from the wedge-like dispersed ions (Yamauchi et al., 2006), and causes a risk of misinterpretation.

There are nearly 720 perigee traversals with CODIF observations of hot ions over the entire traversal by SC-4 during the 2001-2006 period, out of which about 140 traversals are severely contaminated by the radiation belt particles, while nearly 400 traversals are clean enough for the present study. The remaining 180 traversals are partially contaminated by the radiation belt particles but are still possible to use for the present study. Since the contamination is large for low-energy particles due to the time-of-flight type coincidence method, these partially contaminated traversals have two types: one in which all patterns can be identified (nearly 100 traversals) and the other in which only $>\mathrm{keV}$ patterns can be recognized (about 80 traversals). Here, we add the 
first type of partially contaminated traversals to increase the statistics (i.e., total 494 traversals are used).

Another technical problem in taking the statistics is the degradation of the MCP by the accumulated flux over the mission, which makes the weak signatures more difficult to detect in later years. The MCP efficiency decreased by an order of magnitude during the first 4 years even after the bias adjustment (Kistler et al., 2013), and the degraded signalto-noise ratio makes the pattern identification more difficult. Therefore, the MCP degradation may affect the statistics, particularly when we consider the inbound-outbound differences. To minimize this problem, we take a similar but more restrictive selection criterion of the ion signatures than Yamauchi et al. (2006).

For each ion signature, we count the cases only when the entire structure (not the peak) is clearly identified by eye in the energy flux spectrogram of format shown in Fig. 2: we counted ion signatures with about more than $3 \times 10^{5} \mathrm{keV} \mathrm{cm}^{-2} \mathrm{~s}^{-1} \mathrm{str}^{-1} \mathrm{keV}^{-1}$ over the entire structure. The inbound-outbound difference is defined by a change in the intensity of the entire structure by about a factor of 3 using this identification method for the types shown in Figs. $2 \mathrm{~b}-\mathrm{d}$ or by the energy-latitude dispersion direction for the type shown in Fig. 2e. Here, we are not strict in the conjugacy by degree-by-degree because we are interested in the entire structure and because the north-south conjugacy is not strict particularly during winter and summer.

The solar cycle dependence should also be considered because it predicts a decrease in the occurrence frequency of magnetospheric disturbances and electric field after 2004 (e.g., Nakano, 2004). To examine both the MCP degradation effect and the solar cycle effect on the statistics, we examined the bi-annual change of the observation frequency (\%) of (a) the wedge-like dispersed ions, (b) vertical stripes, and (c) short bursts of low-energy ions. As a reference, we also examined (d) the statistics of AL activities during all these traversals. Figure 3 shows the results.

For all three types, the observation frequency drastically decreased after 2004 although the number of substorms did not decrease very much, as shown in Fig. 3d. Therefore, the decrease of the observation frequency of all patterns after 2004 most likely reflects the MCP degradations more than the solar cycle dependence. Considering the increase of the auroral electrojet, AE (AL), activity from the 2001-2002 period to the 2003-2004 period, the MCP degradation is the likely cause of less clear wedge-like dispersed ions during the 2003-2004 period than the 2001-2002 period. Subtracting such a degradation effect, the increase of the vertical stripes during the same period in Fig. $3 b$ should be more drastic in reality. The gradual decrease of the perigee altitude might also cause some effect on the observation, but the observation frequency should not be very much affected because we expect that the spacecraft traverses the same $L$ shell as where these ions are filled.
The rapid decrease of the observation frequency shown in Fig. 3 might cause a different local time distribution between solar maximum (2001-2002) and solar minimum (2006), the latter of which is weighted too little due to the degradation of the instrument. In such a case, using the entire period of 2001-2006 might skew the different local time distributions. To examine this, Fig. 4 shows a comparison of the local time distributions of the wedge-like dispersed ions between the first three years and the last three years. Note that Fig. 4 is made for the entire perigee traversals over both hemispheres, while similar statistics in Yamauchi et al. (2006) are based on one-hemispheric traversals for $2.5 \mathrm{yr}$. Furthermore, the classification in Yamauchi et al. (2006) is not as sub-divided as the present classification. For example, we removed cases without smooth energy-latitude dispersions in Fig. 4. Yet the result for the 2001-2003 period is similar to Fig. 3 of Yamauchi et al. (2006).

Figure 4 demonstrates that, despite the drastic decrease of the observation frequency after 2004 at all LTs, the local time distribution did not change very much between the 20012003 period and the 2004-2006 period. The minor shift of the peak LT from $08 \mathrm{LT}$ to $04 \mathrm{LT}$ might be explained by statistical uncertainty (e.g., binning method and the MCP degradation), and we cannot attribute it to anything at moment. Since the relative profile of the local time distribution is so similar between two periods, we can safely use the entire period data.

Figure 5 shows the local time distributions of the three basic patterns sub-divided by inbound-outbound differences with a criterion of enhancement or decay defined by about a factor of 3 difference in the intensity of the entire structure (category of both large change and some change in Fig. 3). The left panels (Figs. 5a, c, and e) show all sub-categories, and the right panels (Figs. 5b, d, and f) show only enhancing ones. The internal asymmetry in the energy-latitude dispersion direction (Fig. 2e) is also subclassified in Figs. 5a and $b$. The total observation probability is peaked at the late morning sector for the wedge-like dispersed ions and at the midnight sector for the vertical stripes, while the peak in the afternoon for the low-energy bursts is less outstanding than the other patterns.

Both Figs. 3 and 5 demonstrate that, for all the ion signatures, the traversals with significant difference between the inbound and the outbound passes are observed more frequently than cases that are symmetric with respect to the equator, for all periods and at almost all LTs. In particular, a majority of the vertical stripes show significant enhancement during the traversals. This result indicates that the timescale of these phenomena is comparable to the inbound-outbound traversal time (1-2 h).

Figure 5 also shows that the local time distribution for the wedge-like dispersed ions is similar between symmetric cases and cases with significant changes. Also, the fraction of the traversals with significant change over total observations does not show very strong local time dependences for 

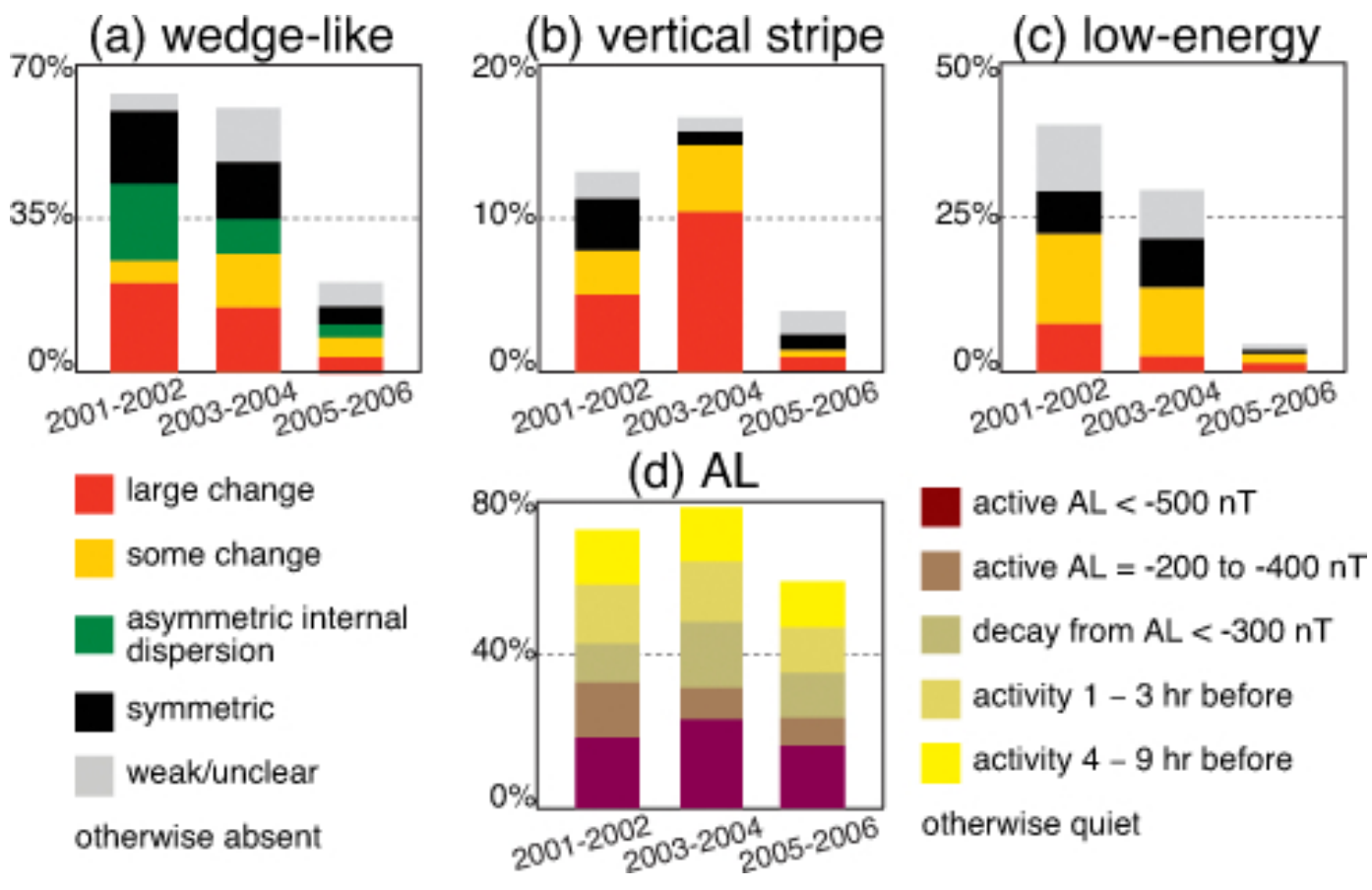

otherwise absent

otherwise quiet

Fig. 3. Observation frequency (\%) of (a) wedge-like dispersed ions, (b) vertical stripes, and (c) short bursts of low-energy ions during Cluster SC-4 perigee traversals, and (d) AL activity during all these traversals, for 2001-2002 (177 traversals), $2003-2004$ (115 traversals), and 2005-2006 (202 traversals). Each ion signature is sub-classified by inbound-outbound difference (large change means one order of magnitude difference in the differential energy flux; some change means more than about a factor of 3 difference in the differential energy flux) or by the energy-latitude dispersion direction of the internal stripes (asymmetric with respect to the equator is shown by green). Symmetry means no significant change in the intensity or energy-latitude dispersion direction. The geomagnetic activity is classified by the current AL activity as well as the hours from the latest AL activity of $|\mathrm{AL}|>200 \mathrm{nT}$.

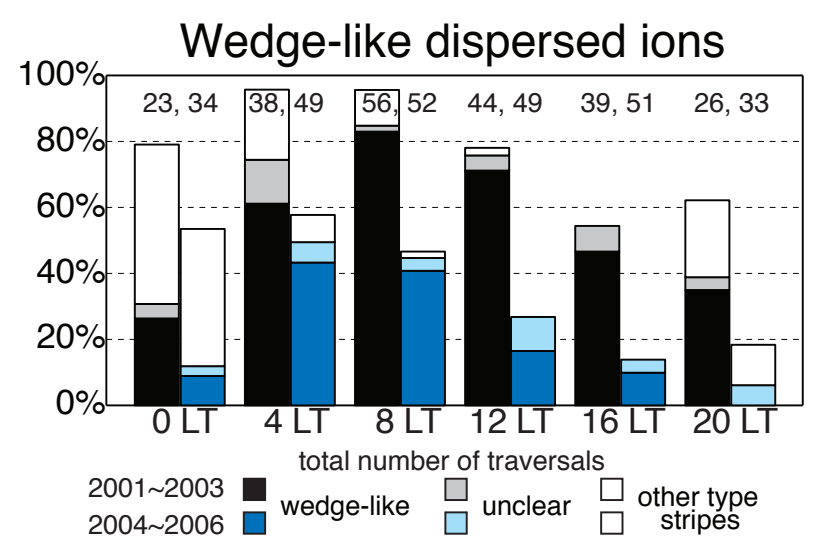

Fig. 4. Observation frequency (\%) of detecting clear wedge-like dispersed ions at different local time (LT) sectors (22-02 LT, 0206 LT, 06-10 LT, 10-14 LT, 14-18 LT, and 18-22 LT) observed by Cluster-4 CIS/CODIF during 2001-2003 and 2004-2006. Number at the top of each bar is the total number of traversals in each $4 \mathrm{~h}$ bin.

the wedge-like dispersed ions and the low-energy bursts. For the vertical stripes and low-energy bursts, however, the local time distribution of the symmetric cases is somewhat different from that of the intensifying cases or total probability.
The local time distribution of the intensifying cases of the low-energy bursts shows a clear peak at noon (Fig. 5f), which is a part of the wide afternoon peak of the total probability (Fig. 5e). The local time distribution of the symmetric cases of the vertical stripes shows a peak at $04 \mathrm{LT}$, where the symmetric cases are found more often than the enhancing cases. This is quite different from the local midnight where the majority are enhancing cases. Although the statistics are not very large, these differences cannot be attributed solely to the statistical error.

The concentration of the vertical stripes, particularly the intensifying ones, to the midnight sector indicates a possible direct link to the substorm activity (McIlwain, 1975; Ebihara et al., 2001). To examine this, Fig. 6 shows statistics of the ion patterns during different geomagnetic conditions using the geomagnetic AL index (minute value). For the indication of "active" conditions, we used an AL threshold of $-300 \mathrm{nT}$ in Fig. 6. After some experimentation, we found that a threshold of $-300 \mathrm{nT}$ gave the clearest contrast between three categories in Fig. $6 \mathrm{~b}$ among the three AL thresholds $-200,-300$, and $-400 \mathrm{nT}$. For the vertical stripes, which are observed most frequently in the local midnight sector, we also show the result for this LT in Fig. 6b. 
(a) Wedge-like dispersed ions

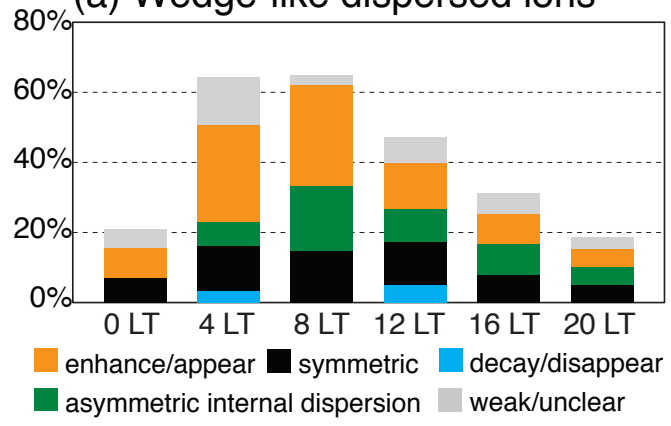

(c) Vertical stripes

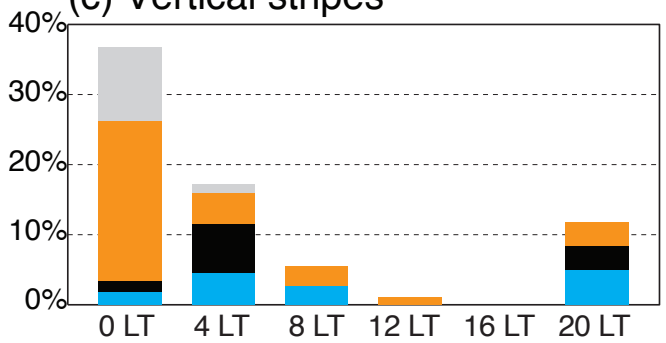

(e) Low-energy bursts

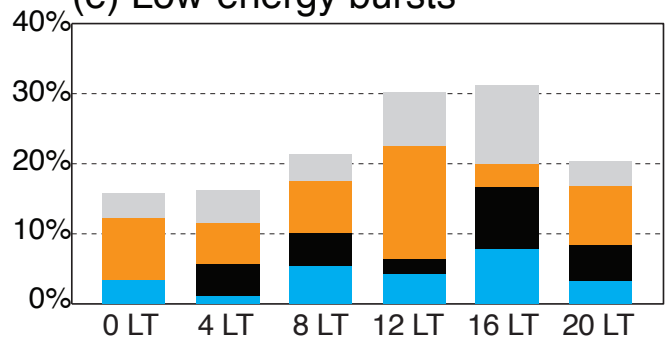

(b) Enhanced cases

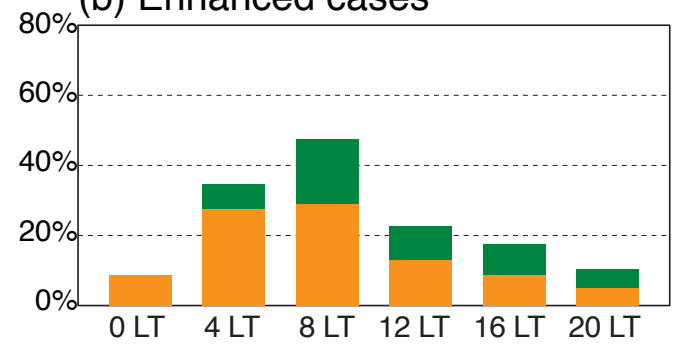

(d) Enhanced cases

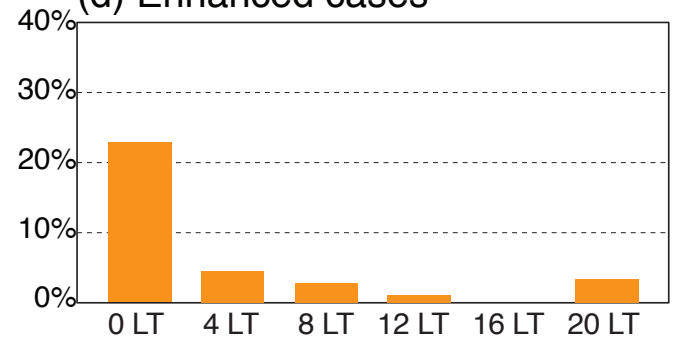

(f) Enhanced cases

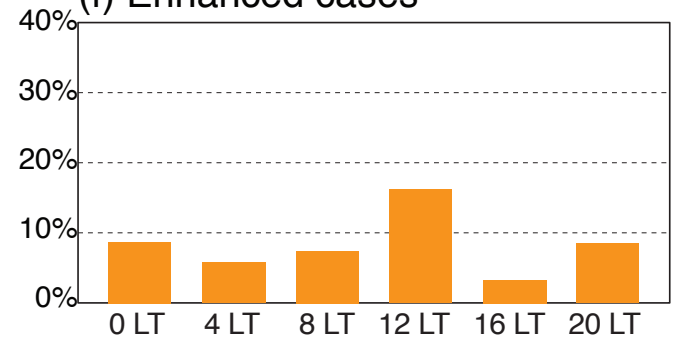

Fig. 5. Observation frequency (\%) of (a)-(b) wedge-like dispersed ions, (c)-(d) vertical stripes, and (e)-(f) short bursts of low-energy ions at different local time (LT) sectors, during Cluster SC-4 perigee traversals during the 2001-2006 period (494 traversals). Traversals with inbound-outbound difference (increase or decrease) are defined by more than about a factor of 3 changes in the differential energy flux. The total number of traversals in each $4 \mathrm{~h}$ bin is 57, 87, 108, 93, 90, and 59, for 22-02 LT, 02-06 LT, 06-10 LT, 10-14 LT, 14-18 LT, and 18-22 LT, respectively.

Figures $6 \mathrm{a}$ and $6 \mathrm{~b}$ demonstrate that the vertical stripes appear as the result of substorm activity mainly in the midnight sector. If the geomagnetic activity is $\mathrm{AL}<-300 \mathrm{nT}$, the vertical stripes appear with more than $60 \%$ probability at local midnight. Figure $6 \mathrm{c}$ demonstrates that, for the wedgelike dispersed ions, their intensification during a traversal is more frequently observed after substorms than during a substorm. This result is consistent with the long-time eastward drift of the wedge-like dispersed ions as demonstrated by the Viking data (Yamauchi and Lundin, 2006). Conversely, internal striping structures with asymmetric energy-latitude dispersion directions are more often observed during substorms than during long quiet periods.

The low-energy burst is observed independent of substorm activity, and even during long quiet periods. This is somewhat different from the statistics of similar structure observed near the equator (e.g., Horwitz and Chappell, 1979).
We made the same plot for local noon where the asymmetric cases are peaked (not shown here), but only a minor association with AL dependence is obtained with a large uncertainty. Thus, a substantial percentage of the low-energy bursts is generated without substorms, while some low-energy bursts are generated during substorms (Yamauchi et al., 2009a). At the moment, we cannot make a general conclusion on the relation between the low-energy burst and substorm activity.

\section{Discussion}

As mentioned in the discussion of Fig. 4, the degradation of MCP as shown in Fig. 3 affects the statistics in such a way that more events are observed in the first three years (2001-2003) than in the following three years (2004-2006) of the Cluster mission. Therefore, the local time distribution (Figs. 5 and 6) mainly reflects the solar maximum condition. 

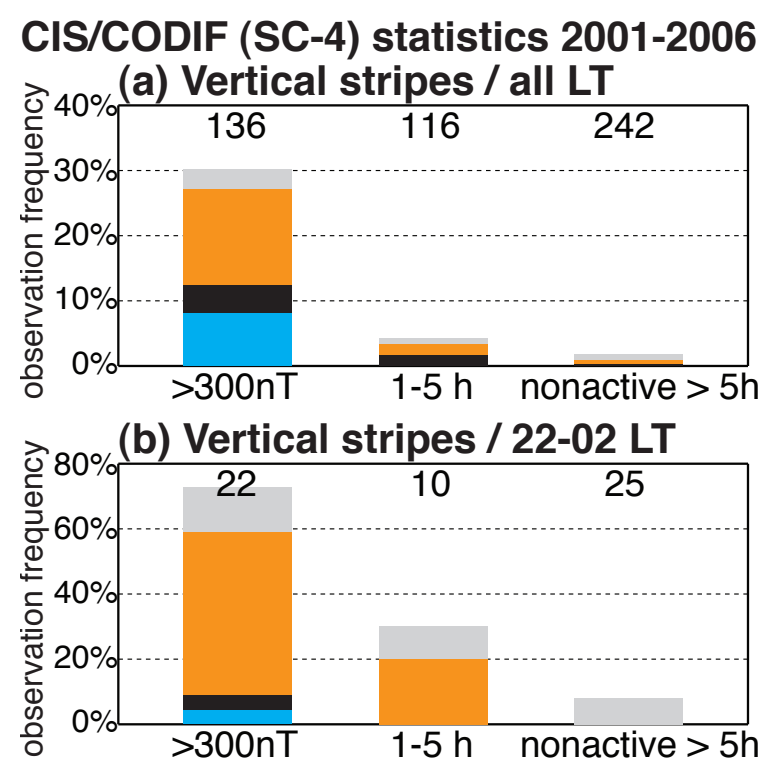

(c) Wedge-like dispersion / all LT
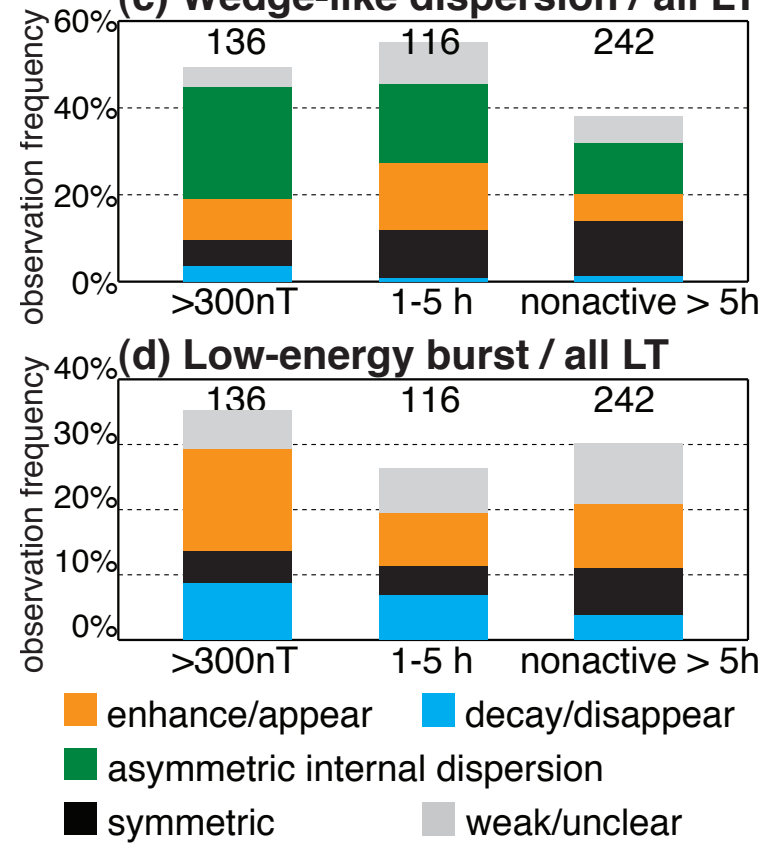

Fig. 6. Observation frequency (\%) of (a) vertical stripes at all LTs, (b) vertical stripes at local midnight, (c) wedge-like structure at all LTs, and (d) short bursts of low-energy ions at all LTs, during Cluster SC-4 perigee traversals during the 2001-2006 period. The observation frequency is obtained for different geomagnetic conditions judged by AL threshold of $-300 \mathrm{nT}$ (minute value), i.e., active period, shortly after active period ( $|\mathrm{AL}|<300 \mathrm{nT}$ for $1-5 \mathrm{~h}$ ), and quiet period ( $|\mathrm{AL}|<300 \mathrm{nT}$ for more than $5 \mathrm{~h}$ ).

Figures 5 and 6 indicate that the vertical stripe is a midnight signature associated with $\mathrm{AL}<-300 \mathrm{nT}$ substorms, while the wedge-like dispersed ions are a dawn signature that appears some hours after a substorm. These results suggest that the vertical structure might be one form of the wedge-like dispersed ions with very short elapsed time (Ebihara et al., 2001). However, if we take the total observation probability, counting both the wedge-like dispersed ions and the vertical stripes, the distribution is still peaked in the morning sector but not the midnight sector as shown in Fig. 4 (see also Yamauchi et al., 2006). Furthermore, some wedge-like dispersed ions are most likely formed as the result of direct filling of low-energy (less than $100 \mathrm{eV}$ ) ions from the ionosphere (Yamauchi et al., 2006; Giang et al., 2009). Therefore, only a fraction of the wedge-like dispersed ions are most likely the result of time-evolved vertical stripes.

The local time distribution is also different between the asymmetric internal dispersion and the wedge-like dispersed ions for both symmetric cases and intensifying cases. This also encourages us to classify the asymmetric internal dispersion as different from the intensifying cases of the wedge-like dispersed ions.

One outstanding result for all three patterns is the high occurrence rate of traversals with significant changes between the inbound and the outbound passes. Among these traversals, intensifying cases dominate over decaying cases for the wedge-like dispersed ions and the vertical stripes, while the intensifying cases and decaying cases show nearly equal observation probability for the low-energy bursts.

The high occurrence rate of intensifying cases at local midnight for the vertical stripes (Fig. 5c) is reasonable because they are directly associated with substorm activity (Fig. 6a), which normally has a timescale of less than $1 \mathrm{~h}$ (i.e., shorter than the Cluster traversal time). The nearly equal observation probabilities between the intensifying cases and decaying cases for the low-energy bursts can be understood by a short lifetime, so that the phenomenon decays quickly when the source mechanism turns off. We should still note that there is some connection between the inbound ion signature and the outbound ion signature because symmetric cases are observed as often as changing cases at around 16LT. This indicates that the lifetime is not very much shorter than the traversal time. Since the pitch-angle distribution of this category is nearly field-aligned in both directions, an ionospheric source is suspected (cf. Quinn and McIlwain, 1979; Horwitz and Chappell, 1979), but no Cluster work has been dedicated to this problem in the inner magnetosphere.

The local time distribution of the enhancing cases in the wedge-like dispersed ions is not intuitively understood because the ion drift at sub-keV range is expected to stagnate near local noon (Ebihara et al., 2001). Although Yamauchi and Lundin (2006) have shown that the drifting speed could be much faster than the model, a numerical work in the future is needed to examine whether the drifting model alone can explain the enhancement within $1-2 \mathrm{~h}$ near local noon or if we need new sources to explain it.

When we examined the correlation between the enhancing cases and geomagnetic activity, we found that the $\mathrm{AL}$ threshold of $-300 \mathrm{nT}$ gave the clearest contrast between the three categories in Fig. $6 \mathrm{~b}$ compared to -200 or $-400 \mathrm{nT}$. 
The wedge-like dispersed ions have less sensitivity in the threshold value. A previous study using Viking satellite data (Yamauchi and Lundin, 2006) gave a similar value: 300$400 \mathrm{nT}$ was the best threshold for hourly AE when examining the wedge-like dispersed ions in the Viking ion data. Therefore, $\mathrm{AL}=-300 \mathrm{nT}$ is a good estimate of the value, beyond which a substorm accompanies formation of ion stripe structures in the inner magnetosphere.

\section{Conclusions}

Using Cluster spacecraft-4 CIS/CODIF data during the 2001-2006 period, we statistically examined the local time distribution of structured trapped ions at the sub- to few $\mathrm{keV}$ range for both the total observation frequency and the ratio of cases that show significant inbound-outbound differences. We particularly examined three basic energylatitude patterns of ions in the inner magnetosphere that are commonly observed in the sub-keV range: (1) wedge-like energy-latitude dispersed structured ions at less than a few $\mathrm{keV}$, (2) short-lived ion stripes with weak dispersion over wide energy ranges $(0.1-10 \mathrm{keV})$, and (3) short-lived lowenergy ion burst (less than a few hundred eV) that are mostly flowing nearly along the geomagnetic field. The differences are defined either by the energy-latitude dispersion direction or by the changes in energy flux of the eye-identified entire ion patterns by about a factor of 3 . Such inboundoutbound differences are attributed to temporal development on the timescale of the traversal when the Cluster orbit was nearly north-south symmetric during the 2001-2006 period. The statistics revealed the following results.

1. The wedge-like dispersed ions are most often observed in the dawn sector (60\% of traversals), which is consistent with the past results (e.g., Yamauchi et al., 2006). The correlation between the wedge-like dispersed ions and the AL activity is consistent with the previous results (Yamauchi and Lundin, 2006; Yamauchi et al., 2009b). However, the high occurrence rate of the enhancing cases at all LTs needs quantitative examination (e.g., by numerical simulation in the future).

2. The short lived ion stripes with weak dispersion over wide energy ranges (vertical stripes) are found predominantly near midnight, where most stripes are significantly enhanced during the traversals and are associated with substorm activities with geomagnetic $\mathrm{AL}<$ $-300 \mathrm{nT}$. Adding this category to the above category does not alter the morning peak of the above category: the vertical stripes may explain only a part of the wedge-like dispersed ions. The local time distribution is also different between the asymmetric internal dispersion and the wedge-like dispersed ions, suggesting that asymmetric internal dispersion is also different from the intensifying cases of the wedge-like dispersed ions.
3. The low-energy bursts are observed at all LTs under all geomagnetic conditions, with a moderate peak in the occurrence rate in the afternoon sector. A large portion of them again show significant enhancement or decay during the traversals. While the afternoon peak is consistent with similar field-aligned warm ions that are observed near the equatorial plane (e.g., Horwitz and Chappell, 1979), the AE dependences are different between the low-energy bursts observed by Cluster and the similar phenomenon in the past.

The statistics also show that intensifying cases dominate over decaying cases for the wedge-like dispersed ions and the vertical stripes, and both the intensifying and decaying cases are nearly comparable for the low-energy bursts. These results indicate significant temporal changes within $1-2 \mathrm{~h}$ in the inner magnetospheric ion populations, and a short lifetime that is comparable to the traversal time (1-2h) for the low-energy bursts.

Correlation studies with the geomagnetic AL activities revealed that the vertical stripes are directly related to substorm activities of $\mathrm{AL}<-300 \mathrm{nT}$, and this relation (occurrence rate of enhancing cases under different $\mathrm{AL}$ conditions) is the clearest when we set the threshold value of AL to $-300 \mathrm{nT}$ instead of $-200 \mathrm{nT}$ or $-400 \mathrm{nT}$. The $-300 \mathrm{nT}$ threshold is also found for the wedge-like dispersed ions. Therefore, AL value of about $-300 \mathrm{nT}$ corresponds to the minimum energy for a substorm that can form the stripe structure (both nondispersed and dispersed).

Acknowledgements. The AE index from 11 stations is provided by WDC-C2 for geomagnetism at Kyoto University. The Cluster project is managed by European Space Agency (ESA). This work is partly supported by Swedish National Space Board (RS). Yamauchi thanks programs for disabled people in Sweden, which have made it possible for him to work.

Topical Editor C. Owen thanks two anonymous referees for their help in evaluating this paper.

\section{References}

Alfvén, H. and Fälthammar, C. G.: Cosmical Electrodynamics, Fundamental Principles, Clarendon, Oxford, 1963.

Ebihara, Y., Yamauchi, M., Nilsson, H., Lundin, R., and Ejiri, M.: Wedge-like dispersion of sub-keV ions in the dayside magnetosphere: Particle simulation and Viking observation, J. Geophys. Res., 106, 29571-29584, 2001.

Ebihara, Y., Ejiri, M., Nilsson, H., Sandahl, I., Grande, M., Fennell, J. F., Roeder, J. L., Weimer, D. R., and Fritz, T. A.: Multiple discrete-energy ion features in the inner magnetosphere: 9 February 1998, event, Ann. Geophys., 22, 1297-1304, doi:10.5194/angeo-22-1297-2004, 2004.

Ejiri, M., Hoffman, R. A., and Smith, P. H.: Energetic particle penetration into the inner magnetosphere, J. Geophys. Res., 85, 653663, 1980. 
Engwall, E., Eriksson, A. I., Cully, C. M., André, M., Torbert, R., and Vaith, T.: Earth's ionospheric outflow dominated by hidden cold plasma, Nature Geoscience, 2, 1, 24-27, doi:10.1038/ngeo387, 2009.

Fennell, J. F., Croley Jr., D. R., and Kaye, S. M.: Low-energy ion pitch angle distributions in the outer magnetosphere: Ion zipper distributions, J. Geophys. Res., 86, 3375-3382, doi:10.1029/JA086iA05p03375, 1981.

Ganushkina, N. Y., Pulkkinen, T. I., Sergeev, V. A., Kubyshkina, M. V., Baker, D. N., Turner, N. E., Grande, M., Kellett, B., Fennell, J., Roeder, J., Sauvaud, J.-A., and Fritz, T. A.: Entry of plasma sheet particles into the inner magnetosphere as observed by Polar/CAMMICE, J. Geophys. Res., 105, 2520525219, doi:10.1029/2000JA900062, 2000.

Giang, T. T., Hamrin, M., Yamauchi, M., Lundin, R., Nilsson, H., Ebihara, Y., Rème, H., Dandouras, I., Vallat, C., BavassanoCattaneo, M. B., Klecker, B., Korth, A., Kistler, L. M., and McCarthy, M.: Outflowing protons and heavy ions as a source for the sub-keV ring current, Ann. Geophys., 27, 839-849, doi:10.5194/angeo-27-839-2009, 2009.

Horwitz, J. L. and Chappell, C. R.: Observations of warm plasma in the dayside plasma trough at geosynchronous orbit, J. Geophys. Res., 84, 7075-7090, doi:10.1029/JA084iA12p07075, 1979.

Kistler, L. M., Mouikis, C. G., and Genestreti, K. J.: In-flight Calibration of the Cluster/CODIF sensor, Geosci. Instrum. Method. Data Syst. Discuss., 3, 221-250, doi:10.5194/gid-3-221-2013, 2013.

Kovrazhkin, R. A., Sauvaud, J.-A., and Delcourt, D. C.: INTERBALL-Auroral observations of $0.1-12 \mathrm{keV}$ ion gaps in the diffuse auroral zone, Ann. Geophys., 17, 734-742, doi:10.1007/s00585-999-0734-9, 1999.

Lennartsson, W., Shelley, E. G., Sharp, R. D., Johnson, R. G., and Balsiger, H.: Some initial ISEE-1 results on the ring current composition and dynamics during the magnetic storm of December 11, 1977, Geophys. Res. Lett., 6, 483-486, 1979.

McIlwain, C. E.: Substorm injection boundaries, in: Magnetospheric Physics, edited by: McCormac, B. M., 143-154, D. Reidel, Hingham, Mass, 1974.

McIlwain, C. E.: Auroral electron beams near the magnetic equator, in: Physics of the Hot Plasma in the Magnetosphere, edited by: Hultqvist, B. and Stenflo, L., 91-112, Springer, New York, 1975.

Nakano, S.: Solar activity dependence of two types of east-west geomagnetic disturbances at mid latitudes, Adv. Polar Upper Atmos. Res., 18, 23-34, 2004.

Newell, P. T. and Meng, C. I.: Substorm introduction of $1 \mathrm{keV}$ magnetospheric ions into the inner plasmasphere, J. Geophys. Res., 91, 11133-11145, 1986.

Olsen, R. C., Shawhan, S.D., Gallagher, D. L., Green, J. L., Chappell, C. R., and Anderson, R. R.: Plasma observations at the Earth's magnetic equator, J. Geophys. Res., 92, 2385-2407, doi:10.1029/JA092iA03p02385, 1987.

Quinn, J. M. and McIlwain, C. E.: Bouncing ion clusters in the Earth's magnetosphere, J. Geophys. Res., 84, 7365-7370, 1979.

Rème, H., Aoustin, C., Bosqued, J. M., Dandouras, I., Lavraud, B., Sauvaud, J. A., Barthe, A., Bouyssou, J., Camus, Th., Coeur-Joly,
O., Cros, A., Cuvilo, J., Ducay, F., Garbarowitz, Y., Medale, J. L., Penou, E., Perrier, H., Romefort, D., Rouzaud, J., Vallat, C., Alcaydé, D., Jacquey, C., Mazelle, C., d'Uston, C., Möbius, E., Kistler, L. M., Crocker, K., Granoff, M., Mouikis, C., Popecki, M., Vosbury, M., Klecker, B., Hovestadt, D., Kucharek, H., Kuenneth, E., Paschmann, G., Scholer, M., Sckopke, N., Seidenschwang, E., Carlson, C. W., Curtis, D. W., Ingraham, C., Lin, R. P., McFadden, J. P., Parks, G. K., Phan, T., Formisano, V., Amata, E., Bavassano-Cattaneo, M. B., Baldetti, P., Bruno, R., Chionchio, G., Di Lellis, A., Marcucci, M. F., Pallocchia, G., Korth, A., Daly, P. W., Graeve, B., Rosenbauer, H., Vasyliunas, V., McCarthy, M., Wilber, M., Eliasson, L., Lundin, R., Olsen, S., Shelley, E. G., Fuselier, S., Ghielmetti, A. G., Lennartsson, W., Escoubet, C. P., Balsiger, H., Friedel, R., Cao, J.-B., Kovrazhkin, R. A., Papamastorakis, I., Pellat, R., Scudder, J., and Sonnerup, B.: First multispacecraft ion measurements in and near the Earth's magnetosphere with the identical Cluster ion spectrometry (CIS) experiment, Ann. Geophys., 19, 1303-1354, doi:10.5194/angeo19-1303-2001, 2001.

Sauvaud, J.-A., Crasnier, J., Mouala, K., Kovrazhkin, R. A. and Jorjio, N. V.: Morning sector ion precipitation following substorm injections, J. Geophys. Res., 86, 3430-3438, 1981.

Sheldon, R. B. and Spence, H. E.: Alfvén boundaries: Noses and zippers, Adv. Space Res., 20, 445-448, 1997.

Vallat, C., Ganushkina, N., Dandouras, I., Escoubet, C. P., Taylor, M. G. G. T., Laakso, H., Masson, A., Sauvaud, J.-A., Rème, H., and Daly, P.: Ion multi-nose structures observed by Cluster in the inner Magnetosphere, Ann. Geophys., 25, 171-190, doi:10.5194/angeo-25-171-2007, 2007.

Woch, J. and Lundin, R: The low-latitude boundary layer at midaltitudes: Identification based on Viking hot plasma data, Geophys. Res. Lett., 20, 979-982, 1993.

Yamauchi, M. and Lundin, R.: Sub-keV ring current ions as the tracer of substorm injection, Ann. Geophys., 24, 355-366, doi:10.5194/angeo-24-355-2006, 2006.

Yamauchi, M., Lundin, R., Eliasson, L., and Norberg, O.: Mesoscale structures of radiation belt/ring current detected by lowenergy ions, Adv. Space Res., 17, 171-174, 1996.

Yamauchi, M., Brandt, P. C., Ebihara, Y., Dandouras, I., Nilsson, H., Lundin, R., Reme, H., Vallat, C., Lindquvist, P.-A., Balogh, A., and Daly, P. W.: Source location of the wedge-like dispersed ring current in the morning sector during a substorm, J. Geophys. Res., 111, A11S09, doi:10.1029/2006JA011621, 2006.

Yamauchi, M., Dandouras, I., Daly, P. W., Stenberg, G., Frey, H. U., Lindqvist, P.-A., Ebihara, Y., Nilsson, H., Lundin, R., Rème, H., André, M., Kronberg, E. A., Balogh, A., and Henderson, M.: Magnetospheric solitary structure maintained by $3000 \mathrm{~km} / \mathrm{s}$ ions as a cause of westward moving auroral bulge at 19 MLT, Ann. Geophys., 27, 2947-2969, doi:10.5194/angeo-272947-2009, 2009a.

Yamauchi, M., Ebihara, Y., Dandouras, I., and Rème, H.: Dual source populations of substorm-associated ring current ions, Ann. Geophys., 27, 1431-1438, doi:10.5194/angeo-27-14312009, 2009b. 